\title{
Suicide Decline and Improved Psychiatric Treatment Status: Longitudinal Survey of Suicides and Serious Suicide Attempters in Tokyo
}

\section{Yasushi Otaka}

Nippon Medical School Hospital

Ryosuke Arakawa

Nippon Medical School Hospital

Ryuichiro Narishige

Nippon Medical School Hospital

Yoshiro Okubo

Nippon Medical School Hospital

Amane Tateno ( $\nabla$ amtateno@nms.ac.jp )

Nippon Medical School Hospital

\section{Research Article}

Keywords: Iongitudinal studies, psychiatric treatment status, serious suicide attempter, suicide death, suicide decline in Japan

Posted Date: January 19th, 2022

DOI: https://doi.org/10.21203/rs.3.rs-1215486/v1

License: (c) (1) This work is licensed under a Creative Commons Attribution 4.0 International License. Read Full License 


\section{Abstract}

\section{Background:}

Connecting individuals who need psychiatric treatment to adequate medical services has been a major strategy for suicide prevention in Japan. Investigating serious suicide attempters admitted to our Critical Care Medical Center (CCM), we aimed to investigate longitudinal changes of psychiatric treatment status in high-risk suicide individuals, and to explore the association between improvement of psychiatric treatment status and suicide decline.

\section{Methods:}

Subjects were enrolled from two periods, 2006 to 2011 and 2012 to 2017. We collected the data of 32,252 suicides in Tokyo from police reports and the data of 942 suicide attempters admitted to CCM from medical records. Data were collected by age and gender for the number of suicide completers, the number of suicide attempters, and the psychiatric treatment rates, respectively, every year.

ANOVA and t-test were performed to examine whether there were differences between the two periods in the number of suicides and attempters. The difference by the two periods in the psychiatric treatment rate were examined using the chi-square test. Additionally, we analyzed the correlation between annual treatment rate and the number of suicide completers by Pearson's correlation coefficients in subgroups which had changed their psychiatric treatment rate.

\section{Results:}

The number of suicide attempters in the age group 20-39 years decreased together with the number of suicides. Psychiatric treatment rates in male attempters aged 20-59 years improved significantly from $48.7 \%$ to $70.6 \%$ and this improvement was correlated with a decrease in suicides. However, psychiatric treatment rates in the elderly, which have the highest number of suicides in both genders, did not improve and remains low.

\section{Conclusions:}

The number of suicide attempters decreased together with suicides in Tokyo. Improvement of psychiatric treatment status in high-risk suicide male adults may have contributed to the reduction of suicides in Tokyo. However, the continuing low rate of psychiatric treatment in the elderly is a pressing issue for future suicide prevention.

\section{Background}

The number of suicides in Japan suddenly increased in 1998, and it remained at over 30,000 per year, with a suicide rate of higher than 24.0 per 100,000 individuals for fourteen consecutive years [1, 2]. "The Basic Act for Suicide Prevention", the basic law against suicide, was established in 2006, and various 
suicide prevention measures were implemented and promoted in a complex and simultaneous manner [3-5]. Consequently, the number of suicides per year started to decrease, falling below 30,000 per year in 2012, with a relatively large decline in the suicide rate from 24.0 in 2011 to 21.8 in 2012 [2]. The suicide rate continued to decrease to 16.0 by 2019 , but the rate in Japan remains the highest among the $\mathrm{G} 7$ countries [2]. It is also of concern that suicides in Japan will possibly increase again due to the COVID-19 pandemic $[6,7]$. Since there are few studies that provide an explanation for the suicide decline in Japan [8], it is necessary to clarify the factors contributing to the decrease in suicides in order to take appropriate suicide prevention measures in the future.

The mental health activity that promotes connecting individuals who need psychiatric treatment with adequate medical services is one of the major strategies in national suicide prevention plans in Japan [2]. As one of the pillars of suicide prevention, the countermeasure of connecting untreated individuals with high suicide risk with adequate psychiatry treatment was taken, thereby increasing the rate of psychiatric care for groups at high risk of suicide. Thus, improvement of the psychiatric treatment rate for high-risk suicide groups may have contributed to the decrease in the number of suicides in Japan. However, the effect of improved psychiatric treatment rates on suicide reduction has not been verified, as it is impossible to examine longitudinal changes in psychiatric treatment rates in suicide cases based on police reports or psychological autopsies in Japan in terms of viability $[1,9,10]$.

Investigation of serious suicide attempters [11] is thought to be an alternative and promising methodology for exploring factors associated with suicidal behaviors [12]. At our hospital, Nippon Medical School (NMS) Hospital, we have a long experience of psychiatric intervention in the Critical Care Medical Center (CCM) for the most physically ill suicide attempters. Our CCM was established in 1977 as the first advanced emergency medical center in Japan. As one of the tertiary emergency medical facilities of the Tokyo Metropolitan Government, the center receives about 1,600 to 1,800 emergency patients per year and about $5 \%$ are suicide attempters. We have reported the results of retrospective observational studies investigating the characteristics of serious suicide attempters in adolescents as well as of gender differences of precipitating factors for suicide attempts [13, 14].

From the annual report by the Fire and Disaster Management Agency (FDMA), the national statistics on ambulance transportation, the number of cases of ambulance transport due to self-harm peaked at 52,630 in 2009 and then continued to decline to 35,377 by 2017 [15]. It is estimated that the decrease in the number of serious suicide attempters is similar to that in the number of suicide death, although there have been no reports on the differences between the two. A survey targeting suicide attempters would possibly confirm their psychiatric treatment status and assist in the investigation of the long-term changes of psychiatric treatment rates in the suicide high-risk group.

In the present study, we aimed to investigate whether the number of serious suicide attempters admitted to CCM of the NMS Hospital decreased in parallel with the number of suicides in Tokyo during the same period. We also aimed to examine whether the psychiatric treatment status of serious suicide attempters improved or not, as well as to explore any association between improvement of psychiatric treatment 
status in serious suicide attempters and a decrease in suicides during the same period and in the same region, that is, in Tokyo.

\section{Methods}

\section{\ Data sampling}

Study subjects were enrolled from the period between January 2006 and December 2017. We collected the number of suicides in the Tokyo area from an open source that is annually reported by the Metropolitan Police Department (MPD) [16]. In this study, we regarded 2012 as the turning point to a decline in suicides in Japan.

The subjects were patients who attempted suicide and required hospitalization at the CCM for at least two days between January 2006 and December 2017. It was considered an actual "suicide attempt" if there was any intent/desire to die in association with the act, which is the same definition as that of the Columbia-Suicide Severity Rating Scale [17]. We collected data from the patients' medical records retrospectively. We judged patients as being "under psychiatric treatment" if they had appointments or a history of psychiatric medical contact within three months before their suicide attempts.

We separated the subjects into groups of two terms, one of 6 years before the decline in suicide death (January 2006 to December 2011), and the other of 6 years after the start of the decline (January 2012 to December 2017). We defined the changes between the two terms as a factor of "period".

Age was divided into four ranges, younger than or equal to 19 years (19 or younger), 20 to 39 years (2039 ), 40 to 59 years (40-59), and equal to or older than 60 (60 or older).

Data were collected by age and gender for the number of suicide death, the number of suicide attempters, and the psychiatric treatment rates, respectively, every year.

\section{\ Statistical analysis}

For the numbers of suicide attempters and suicide death, differences by the factors of "period", "age", and "gender" were examined by analysis of variance (ANOVA). The main effect of the period was examined in terms of whether there was a difference between the number of attempters and the number of suicide death in the two periods before and after suicide reduction. For the items with a significant interaction, $t-$ tests were performed to determine whether there was a difference in the periods of all of the age and gender subgroups.

Differences in the psychiatric treatment rate between gender according to the two periods were examined by chi-square test. In addition, differences in the psychiatric treatment rate between the two periods according to gender, and between these subgroups, were examined by chi-square test, respectively. 
Additionally, we combined subgroups which had changed their psychiatric treatment rate into one group, and we analyzed the correlation between annual treatment rate and the number of suicide death by Pearson's correlation coefficients.

We used a significance level of $p<0.05$ and two-sided probability. $R$ version 4.0.2 statistical package was used for the entire analysis.

\section{Results}

\section{$\nabla$ Changes in suicides in Tokyo}

There were 32,783 suicides in Tokyo during the whole study period. The 531 (2006-2011: 351; 2012-2017: 180) subjects with insufficient information for age were excluded. Finally, the numbers of suicide death included in the analysis were 17,364 (2006-2011) and 14,888 (2012-2017). According to ANOVA, a significant main effect of "period" was recognized, and the number of suicides in Tokyo decreased significantly $(F(1,1)=44.61, p<0.01)$. There were significant interactions between period and age $(F(1,3)=7.31, p<0.001)$ and between period and gender $(F(1,1)=9.13, p=0.003)$. Significant decreases were observed in age and gender subgroups: males aged 20-39 $(t(10)=3.77, p=0.004)$, males aged 40-59 $(t(10)=3.63, p=0.005)$, males aged 60 or older $(t(10)=2.82, p=0.018)$, and females aged $20-39$ $(t(10)=4.21, p=0.002)$ (Figure 1).

\section{\ Changes in serious suicide attempters admitted to CCM}

During the whole study period, 21,271 patients were admitted to the CCM, and the number of suicide attempters included for the analysis was 942. The numbers of suicide attempters in 2006-2011 and 2012-2017 were 573 (5.0\% of 11,452, total CCM inpatients) and 369 (3.8\% of 9,819), respectively. The results of ANOVA showed that there was a main effect of period, and the number of suicide attempters decreased significantly $(F(1,1)=26.20, p<0.001)$. A significant interaction was found between period and age $(F(1,3)=9.05, p<0.001)$ but not between period and gender $(F(1,1)=0.003, p=0.96)$, and a significant decrease was observed only in the subgroup of those aged 20-39 $(t(10)=4.40, p=0.001)$. Further, $t$-tests of changes by age and gender subgroups confirmed a significant decrease only in males aged 20-39 $(t(10)=4.89, p<0.001)$ and females aged 20-39 $(t(10)=2.55, p=0.029)$ (Figure 2).

\section{Q Changes in psychiatric treatment rate in serious suicide attempters}

There was a significant gender difference in the rate of psychiatric treatment. Before the suicide reduction, the rate was higher in females $(69.5 \%$ in females and $44.6 \%$ in males $)\left(X^{2}(1)=35.70, p<0.001\right)$, and after the suicide reduction, the rate was also higher in females $(74.1 \%$ in females and $63.1 \%$ in males) $\left(\chi^{2}(1)=5.01, p=0.025\right)$ (Table 1$)$.

Table 1 Differences in psychiatric treatment rates by gender and period 


\begin{tabular}{|c|c|c|c|}
\hline & \multicolumn{2}{|c|}{ Psychiatric trea tment rate ( trea ted tocal attempters) } & \multirow{2}{*}{$\begin{array}{c}\text { Significance } \\
\text { (Difference by period) }\end{array}$} \\
\hline & $2006-2011$ & $2012-2017$ & \\
\hline $\mathrm{Mal}$ & $44.6 \%(108 / 242)$ & $63.1 \%(89 / 141)$ & $z^{2}(1)=12.19 p<0.001$ \\
\hline Female & $69.5 \%(230 / 331)$ & $74.1 \%(169 / 228)$ & n.s. \\
\hline $\begin{array}{c}\text { Signific ance } \\
\text { (Difference by gender) }\end{array}$ & $x^{2}(1)=35.70$ p 40.001 & $z^{2}(1)=5.01 p=0.025$ & \\
\hline
\end{tabular}

Footnote: The psychiatric treatment rates were defined as the percentages of attempters receiving psychiatric treatment among all attempters.; 2006-2011, 6 years before the decline in suicide death (January 2006 to December 2011); 2011-2017, 6 years after the start of the decline (January 2012 to December 2017)

When changes in psychiatric treatment rates were examined for the periods before and after the decrease in suicides, significant improvements in psychiatric treatment rates were found only in the male groups aged 20-39 (from 55.1-78.3\%, $\chi^{2}(1)=7.52, p=0.006$ ) and 40-59 (from 39.5-65.1\%, $\chi^{2}(1)=9.27, p=0.003$ ) (Table 2). When the rate of psychiatric treatment was examined in the group of adult males aged 20 to 59 , the rate improved from $48.7 \%(97 / 199)$ to $70.6 \%(77 / 109)\left(\chi^{2}(1)=13.74, p<0.001\right)$.

In the groups aged 60 years or older, the rate of psychiatric treatment was low before the decrease in suicides, at $24.3 \%$ in males and $42.9 \%$ in females, and remained low after the decrease in suicides, at $32.0 \%$ in males and $45.7 \%$ in females, with no improvement observed (Table 2).

Table 2 Differences in psychiatric treatment rates among subgroups by period (gender and age subgroups)

\begin{tabular}{|c|c|c|c|}
\hline & \multicolumn{2}{|c|}{ Psychiatric trea ment rate ( treated total a trempters) } & \multirow{2}{*}{$\begin{array}{c}\text { Sigiticance } \\
\text { (Difference by period) }\end{array}$} \\
\hline & $2006-2011$ & 2012-2017 & \\
\hline \multicolumn{4}{|l|}{ Male } \\
\hline 19 or younger & $33.3 \%(2.6)$ & $57.1 \%(47)$ & n.s. \\
\hline $20-39$ & $55.1 \%(65 / 118)$ & $78.3 \%(3646)$ & $x^{2}(1)=7.52 \quad p=0.006$ \\
\hline $40-59$ & $39.5 \%(32.81)$ & $65.1 \%(4 \mathrm{I} / 63)$ & $x^{2}(1)=0.27 \quad p=0.003$ \\
\hline 60 or older & $243 \%(9 / 37)$ & $32.0 \%(8.25)$ & n.s. \\
\hline \multicolumn{4}{|l|}{ Female } \\
\hline 19 or younger & $60.9 \%(14.23)$ & $57.1 \%(8.14)$ & n.s. \\
\hline $20-39$ & $81.6 \%(151 / 185)$ & $84.7 \%(100 / 118)$ & n.s. \\
\hline $40-59$ & $58.0 \%(47.81)$ & $73.8 \%(45 / 61)$ & n.s. \\
\hline 60 or older & $42.9 \%(18.42)$ & $45.7 \%(16 / 35)$ & n.s. \\
\hline
\end{tabular}


Footnote: The psychiatric treatment rates were defined as the percentages of attempters receiving psychiatric treatment among all attempters.; 2006-2011, 6 years before the decline in suicide death (January 2006 to December 2011); 2011-2017, 6 years after the start of the decline (January 2012 to December 2017); 19 or younger, younger than or equal to 19 years; 20-39, 20 to 39 years; $40-59,40$ to 59 years; 60 or older, equal to or older than 60 .

\section{Q Correlation between psychiatric treatment rates in serious suicide attempters and numbers of suicides}

The correlation between psychiatric treatment rates and numbers of suicides was examined in the group of males aged 20-59 years who showed improvement of the psychiatric treatment rate in suicide attempters. There was a significant negative correlation between psychiatric treatment rates and numbers of suicides $(r=-0.59, p=0.042)$ (Figure 3$)$. In other words, improvement in the rate of psychiatric treatment was significantly correlated with the decrease in the number of suicides.

\section{Discussion}

\section{Q Decrease of serious suicide attempters admitted to CCM}

Similar to the decrease in the number of suicides in the Tokyo area, the number of serious suicide attempters who were admitted to the CCM also decreased. There was a significant decrease in the number of suicide attempters especially in the age group of 20-39 years for both genders, just as there was a significant decrease in suicides. However, whereas the number of suicides decreased significantly in males aged 40 and older, the number of suicide attempters did not change significantly in either gender. Regarding such discrepancies, it is known that suicide is more common among middle-aged and older males and attempted suicide is more common among young females in Japan [1, 2]. In this study, as in previous reports, the number of suicides was higher among older adults and more male-dominant, whereas the number of suicide attempters was higher among young adults aged 20-39 and was more female-dominant. Thus, the proportions of males and elderly suicide attempters were smaller than that of suicide death, so there is a possibility that we could not confirm the decrease in the number of suicide attempters among males over 40 years old, where a decrease in the number of suicides was observed.

\section{Improvement of psychiatric treatment rate among serious suicide attempters}

The purpose of the present study was to examine whether psychiatric treatment rates improved in a highrisk suicide population. Comparing psychiatric treatment rates of serious suicide attempters admitted to CCM before and after a decrease in suicides, we confirmed a significant improvement in psychiatric treatment rates from $48.7-70.6 \%$ in the group of males aged $20-59$ years. In contrast, no significant improvement was observed in the female group, but this may have been due to their originally high rate of psychiatric treatment. Psychological autopsy studies in Japan reported that $44.1 \%$ to $50 \bigotimes$ of suicide death were treated by psychiatrists $[9,10]$. Considering our present results, the psychiatric treatment rate of serious suicide attempters may be higher than that of suicide death, and the status of psychiatric treatment for a high-risk suicide population may improve during the period of suicide decline. 


\section{Improvement in psychiatric treatment rate and a decrease in the number of suicides}

Interestingly, in the group of males aged 20-59 years, improvement in psychiatric treatment rates was correlated with a decrease in the number of suicides during the same period. This result suggests that improved psychiatric treatment rates among males in this age group may have contributed to the reduction in the number of suicides in Tokyo. There is considerable evidence that measures for connecting untreated populations in need of treatment with psychiatric care are effective in preventing suicide $[18,19]$. Several psychiatric treatments, such as pharmacotherapy and cognitive behavioral therapy, have been shown to be effective in preventing suicidal behavior [20-24]. However, the trend toward higher rates of psychiatric consultation among serious suicide attempter is paradoxical, as it indicates that just only leading to psychiatric consultation is limited in its effectiveness in preventing suicidal behavior. Negative data on psychiatric consultation is also present, pointing to the need to explore alternatives to traditional methods of suicide prevention intervention in the future [25]. It will also be important to collaborate with appropriate support organizations to provide support to patients at high risk of suicide who have been referred to the hospital.

Together with the results of this study, there is a possibility that suicidal behavior decreased with improvement in the psychiatry treatment rate. On the other hand, the elderly group with the highest number of suicides in both males and females had the lowest rate of receiving psychiatric treatment, and they showed no improvement in their psychiatric treatment rate. Elderly population is a group at risk associated with suicide, such as living alone, social isolation associated with COVID-19, and physical illness [25]. These results indicate that people at high risk of suicide in the elderly population may be receiving insufficient psychiatric care, and this is an issue that may require urgent attention.

Limitations of the current study include the fact that we used suicide death data from the whole area of Tokyo. Although suicide attempters are transported to our emergency department from a broad area, this does not cover the whole Tokyo area. So, these study fields do not exactly match. Our study was conducted as a single-site survey. This will also be a limitation of the data representativeness when it is used as a reference for taking suicide countermeasures in the future. Thus, surveys in a broad area and multicenter collaborative studies with large numbers of subjects will be necessary to solve these limitations. In this survey, although we focused on the relationship between the psychiatric treatment rate and the number of suicide death, the contributions of other psychosocial factors and economic factors that have been indicated as also being related to suicide were not examined.

\section{Conclusions}

In conclusion, the number of serious suicide attempters admitted to the CCM decreased together with the number of suicides in Tokyo. Especially in the age group of 20-39 years, the number of serious suicide attempters of both genders decreased, as did the number of suicides. The results of this study confirmed a significant improvement in psychiatric treatment rates in the severe suicide attempt group of males 
aged 20-59 years. Furthermore, improvement in the psychiatric treatment rate was correlated with a decrease in the number of suicides of the same gender and age in the community.

These results suggest that the improvement of psychiatric treatment rates in high-risk suicide groups may have contributed to the reduction of suicides in Tokyo. However, the rate of psychiatric treatment in the elderly group, which has the highest number of suicides among both males and females, has remained low and has not improved, suggesting a future issue needing to be addressed for suicide prevention.

\section{Abbreviations}

ICD: International Statistical Classification of Diseases and Related Health Problems

\section{Declarations}

\section{Consent for publication}

Not applicable.

\section{Availability of data and materials}

The datasets generated and/or analysed during the current study are not publicly available due to the used data protection declaration, but are available from the corresponding author on reasonable request.

\section{Competing interests}

Author Yoshiro Okubo has received grants or speaker's honoraria from Dainippon Sumitomo Pharma, Otsuka, Eli Lilly, Yoshitomi, and Meiji within the past 3 years. Author Ryosuke Arakawa has received speaker's honoraria from Dainippon Sumitomo Pharma and Pfizer within the past 3 years. The remaining authors declare no conflict of interest.

\section{Funding}

Not applicable.

\section{Authors' contributions}

YOt and RN designed the study and wrote the protocol. YOt and RN contributed the acquisition of the data and clinical review. YOt, Yok, and RA analyzed the data. YOt and YOk wrote the first draft of the manuscript. RA, RN, YOk, and AT contributed to the modification of the manuscript. All authors contributed to and have approved the final manuscript.

\section{Acknowledgements}


We thank Dr. Takao Ito, Dr. Yoshitaka Kawashima, Mr. Ken Yamamoto, Mr. Rui Ishida, Mr. Taiki Fujimoto, and all the CCM medical staff of NMS hospital for their collaboration in data collection, and Dr. Daisuke Takagi for his suggestions regarding statistical analysis.

\section{Ethical statement}

Informed consent was not taken as only anonymised information was used in data sampling. The research subjects were presented with an explanation of the study objectives, methods and publication of results, and were guaranteed the opportunity to refuse participation. The present study was approved by the Ethics Committee of Nippon Medical School Hospital (B-2020-113) and conforms to the provisions of the Declaration of Helsinki.

\section{References}

1. Ministry of Health $L$ and W. Statistics of suicide victims in Japan in 2019. 2020, [https://www.npa.go.jp/safetylife/seianki/jisatsu/R02/R01_jisatuno_joukyou.pdf]

2. Ministry of Health $L$ and W. White paper on suicide prevention in Japan. 2020, [https://www.mhlw.go.jp/wp/hakusyo/jisatsu/19/index.html]

3. Takeshima T, Yamauchi T, Inagaki M, Kodaka M, Matsumoto T, Kawano K, et al. Suicide prevention strategies in Japan: A 15-year review (1998-2013). Journal of Public Health Policy. 2015; 36: 52-66.

4. Sawada Y, Ueda M, Matsubayashi T. Economic Analysis of Suicide Prevention: Towards EvidenceBased Policy-making. forthcoming, Springer; 2017. p. 33-68.

5. Motohashi Y. Suicide in Japan. The Lancet. 2012; 379: 1282-3.

6. Tanaka T, Okamoto S. Increase in suicide following an initial decline during the COVID-19 pandemic in Japan. Nature Human Behaviour. 2021; 5: 229-38.

7. Sakamoto H, Ishikane M, Ghaznavi C, Ueda P. Assessment of Suicide in Japan During the COVID-19 Pandemic vs Previous Years. JAMA network open. 2021; 4: e2037378.

8. Nakanishi M, Endo K, Ando S, Nishida A. The impact of suicide prevention act (2006) on suicide in japan. CRISIS. 2019; 41: 24-31.

9. Kodaka M, Matsumoto T, Takai M, Yamauchi T, Kawamoto S, Kikuchi M, et al. Exploring suicide risk factors among Japanese individuals: The largest case-control psychological autopsy study in Japan. Asian Journal of Psychiatry. 2017; 27: 123-6.

10. Hirokawa S, Matsumoto T, Katsumata Y, Kitani M, Akazawa M, Takahashi Y, et al. Psychosocial and psychiatric characteristics of suicide completers with psychiatric treatment before death: A psychological autopsy study of 76 cases. Psychiatry and Clinical Neurosciences. 2012; 66: 292-302.

11. Beautrais AL. Suicides and serious suicide attempts: Two populations or one? Psychological Medicine. 2001; 31: 837-45.

12. Gvion Y, Levi-Belz Y. Serious suicide attempts: Systematic review of psychological risk factors. Frontiers in Psychiatry. 2018;9 MAR:1-17. 
13. Narishige R, Kawashima Y, Otaka Y, Saito T, Okubo Y. Gender differences in suicide attempters: A retrospective study of precipitating factors for suicide attempts at a critical emergency unit in Japan. BMC Psychiatry. 2014; 14: 1-7.

14. Kawashima Y, Ito T, Narishige R, Saito T, Okubo Y. The characteristics of serious suicide attempters in Japanese adolescents- comparison study between adolescents and adults. BMC Psychiatry. 2012, 12:191.

15. Fire and Disaster Management Agency. Kyukyu-kyuujono genkyou. 2018, [https://www.fdma.go.jp/publication/rescue/post-2.html]

16. Metropolitan Police Department. Keishichou no toukei. 2018, [https://www.keishicho.metro.tokyo.jp/about_mpd/jokyo_tokei/tokei/index.html]

17. Posner K, Brown GK, Stanley B, Brent DA, Melvin GA, Greenhill L, et al. The Columbia-Suicide Severity Rating Scale: Initial Validity and Internal Consistency Findings From Three Multisite Studies With Adolescents and Adults. American Journal of Psychiatry. 2011; 168: 1266-77.

18. Oyama H, Sakashita T. Community-based screening intervention for depression affects suicide rates among middle-aged Japanese adults. Psychological Medicine. 2017; 47: 1500-9.

19. Rutz W, von Knorring L WJ. Long-term effects of an educational program for general practitioners given by the Swedish Committee for the Prevention and Treatment of Depression. Acta Psychiatrica Scandinavica. 1992; 85: 83-8.

20. Cipriani A, Hawton K, Stockton S, Geddes JR. Lithium in the prevention of suicide in mood disorders: Updated systematic review and meta-analysis. BMJ (Online). 2013; 347: 1-13.

21. D'Anci KE, Uhl S, Giradi G, Martin C. Treatments for the prevention and management of suicide. Annals of Internal Medicine. 2019; 171: 334-42.

22. Søndergård L, Kvist K, Lopez AG, Andersen PK KLV. Temporal changes in suicide rates for persons treated and not treated with antidepressants in Denmark during 1995-1999. Acta Psychiatrica Scandinavica. 2006; 114: 168-76.

23. Tarrier N, Taylor K GP. Cognitive-behavioral Interventions to Reduce Suicide Behavior: A Systematic Review and Meta-Analysis. Behav Modif. 2008; 32: 77-108.

24. Kawanishi C, Aruga T, Ishizuka N, Yonemoto N, Otsuka K, Kamijo Y, et al. Assertive case management versus enhanced usual care for people with mental health problems who had attempted suicide and were admitted to hospital emergency departments in Japan (ACTION-J): a multicentre, randomised controlled trial. Lancet Psychiatry. 2014; 1: 193-201.

25. Harada K, Eto N, Honda Y, Kawano N, Ogushi Y, Matsuo M, et al. A comparison of the characteristics of suicide attempters with and without psychiatric consultation before their suicidal behaviours: $\mathrm{A}$ cross-sectional study. BMC Psychiatry. 2014; 14.

26. Zalsman G, Stanley B, Szanto K, Clarke DE, Carli V, Mehlum L. Suicide in the Time of COVID-19: Review and Recommendations. Archives of Suicide Research. 2020; 24: 477-82.

\section{Figures}




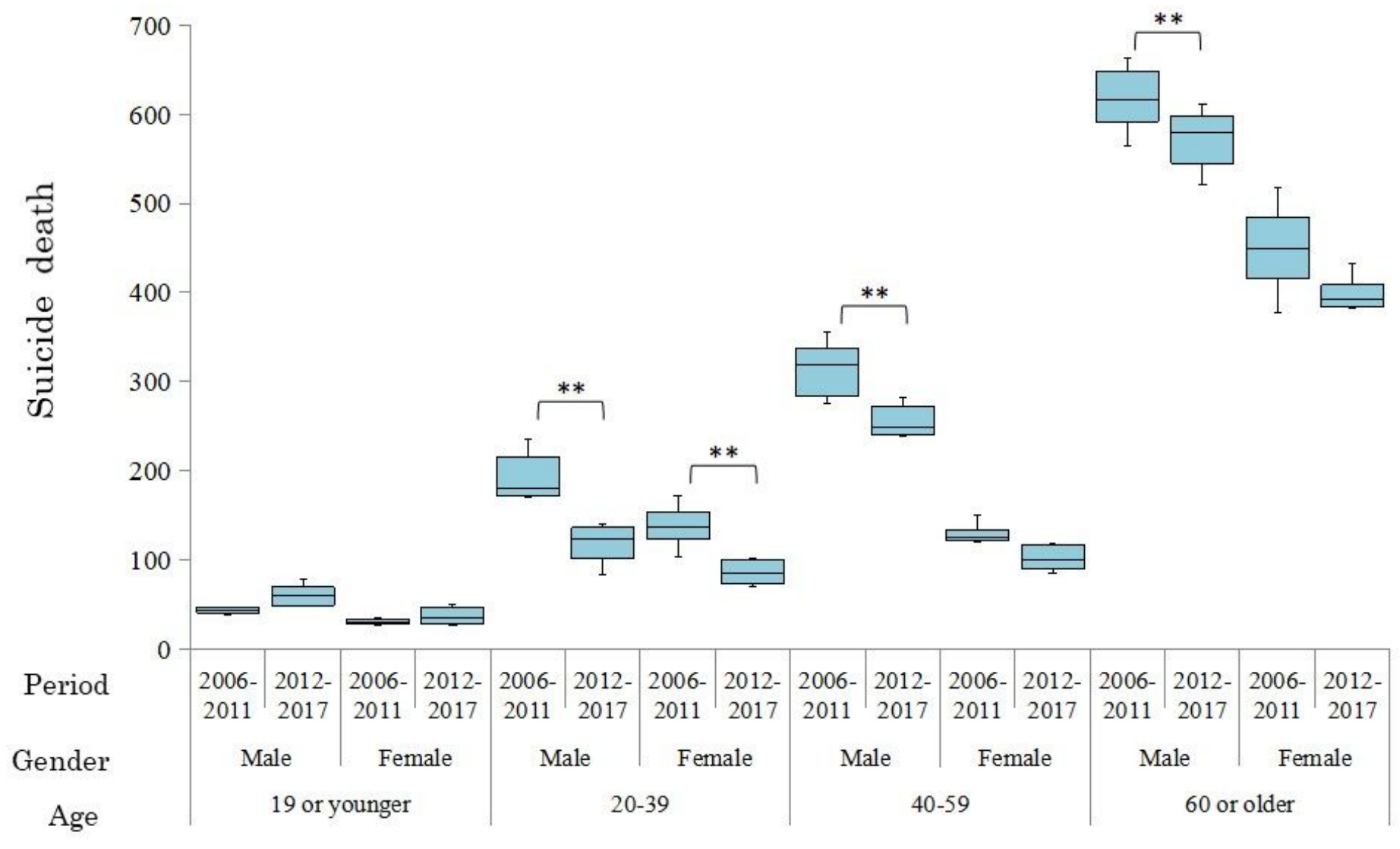

\section{Figure 1}

Changes in the number of suicides in Tokyo by period (age and gender subgroups)

Footnote: $1 .{ }^{*}<.01 ; *<.05$; 2 . Period: 2006-2011, 6 years before the decline in suicide death (January 2006 to December 2011); 2011-2017, 6 years after the start of the decline (January 2012 to December 2017); 3. Age: 19 or younger, younger than or equal to 19 years; 20-39, 20 to 39 years; $40-59,40$ to 59 years; 60 or older, equal to or older than 60. 


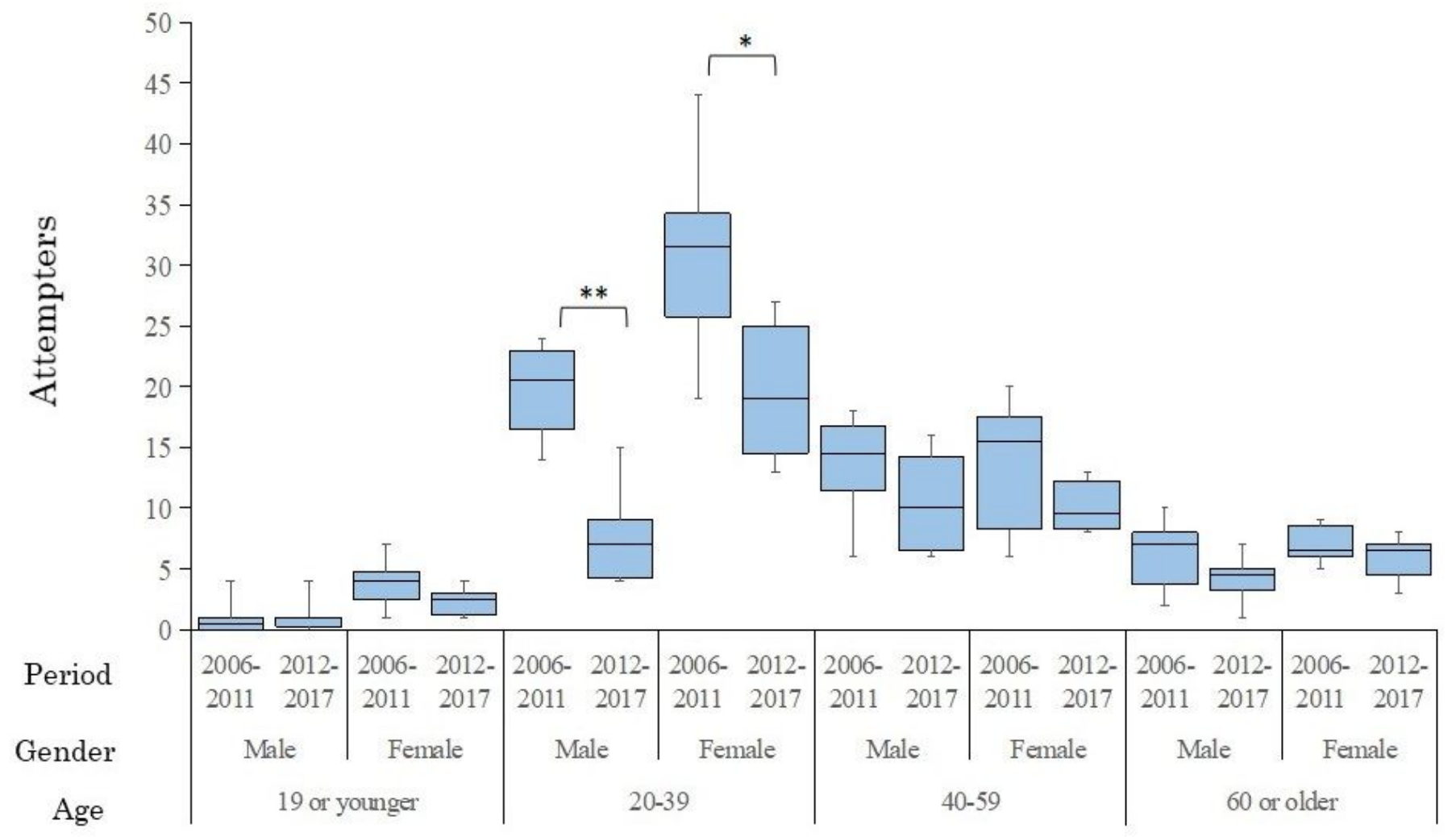

Figure 2

Changes in the number of suicide attempters in CCM by period (age and gender subgroups)

Footnote: $1 .{ }^{*}<.01 ; *<.05 . ; 2$. Period: 2006-2011, 6 years before the decline in suicide death (January 2006 to December 2011); 2011-2017, 6 years after the start of the decline (January 2012 to December 2017); 3. Age: 19 or younger, younger than or equal to 19 years; 20-39, 20 to 39 years; $40-59,40$ to 59 years; 60 or older, equal to or older than 60 . 


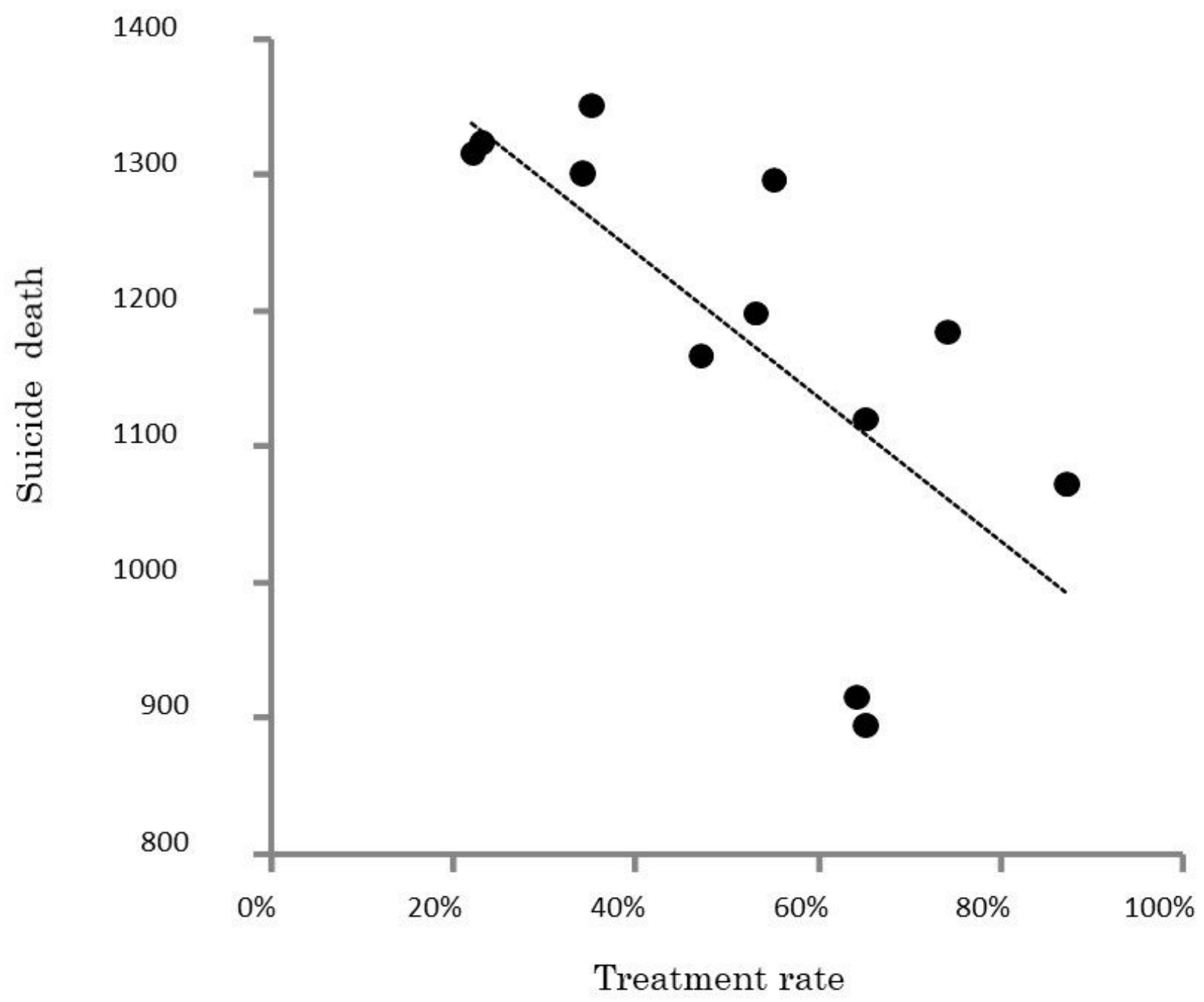

Figure 3

Yearly scatter plot of psychiatric treatment rate and the number of suicide death (males 20-59)

Footnote: The psychiatric treatment rates were defined as the percentages of attempters receiving psychiatric treatment among all attempters of males aged 20-59. 